\title{
Asociación temporal entre la circulación de virus respiratorios y hospitalizaciones por enfermedad de Kawasaki
}

\section{Temporal association between the circulation of respiratory viruses and hospitalizations due to Kawasaki disease}

Daniela Aguirre', Jaime Cerda², Cecilia Perret ${ }^{1}$, Arturo Borzutzky ${ }^{1}$ y Rodrigo Hoyos-Bachiloglu ${ }^{1}$

'Departamento de Enfermedades Infecciosas e Inmunología Pediátrica, División de Pediatría, Escuela de Medicina, Pontificia Universidad Católica de Chile.

${ }^{2}$ Departamento de Salud Pública, Escuela de Medicina, Pontificia Universidad Católica de Chile.

Los autores declaran no tener conflictos de interés.

Estudio sin financiamiento, realizado en la Facultad de Medicina de la Pontificia Universidad Católica de Chile.

Recibido: 13 de marzo de 2020 (segunda versión: 27 de enero de 2021) / Aceptado: 6 de febrero de 2021

\section{Resumen}

Introducción: La etiología de la enfermedad de Kawasaki (EK) es desconocida, planteándose que infecciones virales la gatillan en pacientes susceptibles. Objetivo: Estudiar la asociación temporal entre la circulación de virus respiratorios y hospitalizaciones por EK en la Región Metropolitana (RM), Chile, entre 2010-2017. Metodología: Estudio ecológico retrospectivo de casos de EK en pacientes bajo 18 años de edad, en base a egresos hospitalarios. La circulación de virus se analizó mediante el reporte de la red de vigilancia metropolitana. Se utilizaron promedios móviles para EK (PMEK) y virus respiratorios (PMVR). Resultados: Se registraron 14.902 casos de infecciones virales respiratorias entre 2010-2017. Se observó correlación directa entre PMVR-virus respiratorio sincicial (VRS) de un mes y año y PMEK del mes subsiguiente (coeficiente de correlación $(\rho)=+0,441$; $\mathrm{p}<0,001$ ), y una asociación similar para PMVR-influenza A (FLU A) $(\rho=+0,362 ; p<0,001)$. PMVR-influenza B (FLU B) y PMVRmetapneumovirus (MPV) presentan correlaciones directas con PMEK ( $\rho=+0,443 ; p<0,001$ у $\rho=+0,412 ; p<0,001$, respectivamente), siendo contemporáneo en mes y año con EK para FLU B, mientras que MPV presenta un desfase de un mes entre PMVR y PMEK. Conclusión: Existe correlación temporal directa entre la circulación de VRS, FLU A, FLU B y MPV con EK en niños de la RM, Chile.

Palabras clave: enfermedad de Kawasaki; etiología; epidemiología; Región Metropolitana, virus respiratorios; virus respiratorio sincicial; influenza; metapneumovirus.

\begin{abstract}
Background: The etiology of Kawasaki disease (KD) is unknown. It is believed that viral infections could trigger the disease in susceptible patients. Aim: To study the temporal association between the circulation of respiratory viruses and KD hospitalizations in the Metropolitan Region (MR), Chile, between 2010-2017. Methods: Ecologic study consisting of a review of KD cases in children under 18 years of age based on hospital discharges. The circulation of respiratory viruses was analyzed using the report of the metropolitan surveillance network. Moving averages for KD (MAKD) and respiratory viruses (MARV) were used. Results: 14,902 cases of respiratory virus infections were recorded between 2010-2017. A direct correlation was found between MARV-respiratory syncytial virus (RSV) of one month and year and MAKD of the subsequent month (correlation coefficient $(\rho)=+0.441$; $\mathrm{p}<0.001)$. A similar association was found for MARV-influenza A (FLU A) $(\rho=+0.362 ; p<0.001)$. MARV-influenza B (FLU B) and MARV-metapneumovirus (MPV) had direct correlations with MAKD ( $\rho=+0.443 ; p<0.001$ and $\rho=+0.412 ; p<0.001$, respectively), being FLU B contemporary in month and year with KD, and MPV presenting a one-month lag. Conclusion: There is a direct temporal correlation between RSV, FLU A, FLU B and MPV circulation and $\mathrm{KD}$ in children from RM, Chile.

Keywords: Kawasaki disease; etiology; epidemiology; Metropolitan Region, respiratory viruses; respiratory syncytial virus; influenza; metapneumovirus.
\end{abstract}

\footnotetext{
Correspondencia a:

Rodrigo Hoyos Bachiloglu

rhoyos@med.puc.cl
} 
de las infecciones virales y el desarrollo de EK a nivel poblacional. Por una parte, el reporte de Harnden y cols. no encontró una asociación temporal entre virus respiratorios y EK en población pediátrica de Inglaterra ${ }^{15}$, mientras que Kim y cols. reportaron recientemente la existencia de ésta en población corean $\mathrm{a}^{16}$. Adicionalmente, la aparición de nuevos agentes infecciosos como el SARS-CoV-2, capaces de desencadenar cuadros clínicos muy similares a la EK en pacientes pediátricos, aportan nueva evidencia en favor de un probable gatillante infeccioso en la $\mathrm{EK}^{17,18}$.

El objetivo de este estudio es evaluar, en base a un diseño ecológico, una posible asociación temporal entre la circulación de virus respiratorios y hospitalizaciones por EK. Un objetivo secundario es actualizar los datos epidemiológicos de EK en Chile hasta el año 2017.

\section{Materiales y Métodos}

En base a un diseño de tipo ecológico, se realizó una revisión de la base de datos de egresos hospitalarios del Departamento de Estadísticas e Información de Salud (DEIS) del Ministerio de Salud de Chile (MINSAL), entre los años 2008 y 2017. Se consideró para el estudio aquellos casos codificados como EK (Código ICD-10 M30.3) en pacientes bajo 18 años de edad. La unidad de análisis de la base de datos utilizada es hospitalizaciones. Las tasas de internaciones por EK fueron calculadas por cada 100.000 habs. bajo 5 años. Para el análisis de distribución geográfica se consideró: zona norte (regiones XV-Arica y Parinacota, Regiones I-Tarapacá hasta V-Valparaíso), zona centro (Región Metropolitana) y zona sur (desde la Región VI-Del Libertador Bernardo O”Higgins hasta la Región XII-Magallanes-Antártica Chilena y Región XIV-De los Ríos).

Para el análisis de la circulación de virus respiratorios se utilizaron los datos de la red de vigilancia metropolitana de virus respiratorios en la Región Metropolitana (RM) desde el año 2010 al 2017. Esta red vigila la circulación de los virus respiratorios en Santiago, Osorno y Puerto Montt y se publica en virus.uc.cl; considerando pacientes de toda edad, tanto ambulatorios como hospitalizados y recopila información de centros centinela en las ciudades previamente mencionadas (Servicio de Urgencia del Hospital Clínico de la Pontificia Universidad Católica (PUC), Unidad de Pediatría del Centro Médico San Joaquín (PUC), Laboratorios de Virología PUC, Consultorio $\mathrm{N}^{\circ} 5$ del Servicio de Salud Metropolitano Central, Clínica Alemana de Santiago, Hospital de Enfermedades Infecciosas Dr. Lucio Córdova, Hospital Puerto Montt, Hospital Base San José de Osorno, Unidad de Epidemiología del Servicio de Salud Metropolitano Central, Departamento de Epidemiología MINSAL, Facultad de Medicina de la Universidad del Desarrollo y Exámenes de Laboratorio 
S.A. (Integramédica). Para los fines de este estudio se consideraron sólo los datos recopilados en centros pertenecientes a la Red de Salud UC-Christus en pacientes bajo 5 años. El diagnóstico virológico se realizó mediante inmunofluorescencia directa (IFD) o técnica de reacción de polimerasa en cadena (RPC). Los virus detectados en esta red de vigilancia fueron: virus respiratorio sincicial (VRS), influenza A (FLU A), influenza B (FLU B), adenovirus (ADV), rinovirus (RV), parainfluenza 1 (PIV1), parainfluenza 2 (PIV2), parainfluenza 3 (PIV3), parainfluenza 4 (PIV4), metapneumovirus (MPV), bocavirus, coronavirus y enterovirus.

Cinco semanas epidemiológicas no fueron consideradas en el análisis debido a falta de información, principalmente edad de los pacientes (semana epidemiológica del 1 al 4 de agosto de 2013, 3 al 18 de junio de 2013, 28 de marzo a 3 de abril de 2016, 15 al 21 de mayo de 2016 y del 8 al 14 de mayo de 2016). En aquellas semanas epidemiológicas en que ocurría interposición entre meses (final de uno e inicio de otro) se consideraron los datos para análisis estadístico dentro del mes que finalizaba, a excepción de diciembre, donde fueron considerados en el mes de enero.

El presente estudio fue aprobado por el Comité Ético Científico de la Pontificia Universidad Católica de Chile.

\section{Análisis estadístico}

Con el objetivo de estabilizar fluctuaciones mayores en los datos, se calculó el promedio móvil de los casos de EK (PMEK) y promedio móvil de infección por virus respiratorios (PMVR) para cada mes desde el año 2010 a 2017 (94 meses). Para evaluar la asociación entre casos de EK y casos de infección por virus respiratorios se calculó el coeficiente de correlación de Spearman ( $\rho$ ) y su respectivo valor $\mathrm{p}$. Se consideraron en el presente estudio aquellos virus de mayor circulación, a saber, VRS, FLU A, FLU B, ADV, RV, PIV1, PIV3 y MPV.

El cálculo de correlación entre PMEK y PMVR se hizo considerando tres escenarios posibles: 1) correlación entre PMVR y PMEK para un mismo mes y año (EK0); 2) correlación entre PMVR de un mes y año, y PMEK del mes siguiente (EK1), y 3) correlación entre PMVR de un mes y año, y PMEK del mes subsiguiente (EK2). Por su parte, los cambios temporales en incidencia de EK fueron analizados mediante regresión lineal simple. Para el análisis estadístico se utilizó Python, aplicaciones Excel y el programa estadístico SPSS (Statistical Package for the Social Sciences) versión 23 (IBM Corp., Armonk, NY).

\section{Resultados}

Durante el período comprendido entre 2008 y 2017 se registraron 1.512 hospitalizaciones por EK en pacientes bajo 18 años de edad a lo largo del país. Las características demográficas de estos egresos se muestran en la Tabla 1. La edad mediana de presentación fue de 2 años, con una predominancia masculina (61\%). Un $85 \%$ de los casos correspondió a pacientes bajo 5 años, sin registrarse muertes intrahospitalarias por EK durante los años estudiados. El promedio de estadía hospitalaria fue de 7 días para pacientes bajo 5 años (rango de-110). El análisis de la distribución de los casos a lo largo del año mostró un mayor número de hospitalizaciones por EK entre los meses de agosto y octubre, meses que concentran el 30\% de los casos del período en pacientes bajo 5 años.

La tasa nacional de hospitalización por EK entre 2008 y 2017 fue de 10,5 casos por 100.000 niños bajo 5 años (IC95\% 9,9-11,1) (Figura 1). El análisis de la evolución temporal de las hospitalizaciones por EK no demostró cambios significativos desde el año $2008(\beta=-0,18$, IC95\% - 0,43 a 0,$8 ; p=0,15$ ). El $45 \%$ de las hospitalizaciones fueron registradas en la RM, la que presenta la tasa más alta de internación entre los años 2008 y 2017 (12 casos por 100.000 niños bajo 5 años, IC95\% 10,2-13,8). $\mathrm{Al}$ analizar la distribución de los casos en la RM según servicios de salud, se observa que el Servicio de Salud Metropolitano Oriente concentró el mayor porcentaje de casos (Figura 2).

\begin{tabular}{|c|c|}
\hline & n (\%) \\
\hline Hombres & $923(61)$ \\
\hline \multicolumn{2}{|l|}{ Edad } \\
\hline$<1$ año & $377(25)$ \\
\hline 1-4 años & $913(60)$ \\
\hline 5-9 años & $189(13)$ \\
\hline 10-17 años & $33(2)$ \\
\hline \multicolumn{2}{|l|}{ Previsión } \\
\hline FONASA & $1.012(67)$ \\
\hline ISAPRE & $397(26)$ \\
\hline Otra & $103(7)$ \\
\hline \multicolumn{2}{|c|}{ Distribución geográfica } \\
\hline Norte & $282(19)$ \\
\hline RM & $683(45)$ \\
\hline Sur & $547(36)$ \\
\hline
\end{tabular}




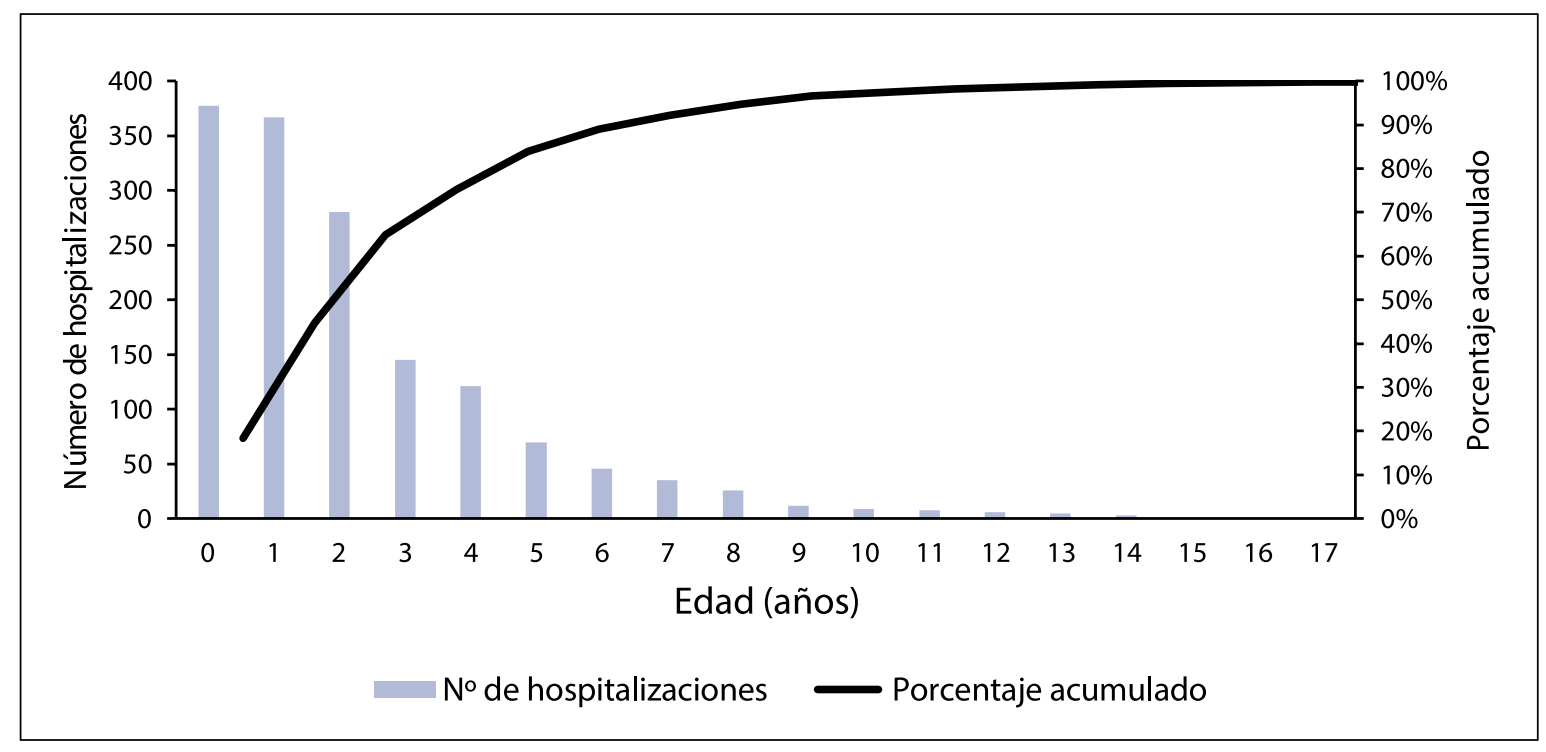

Figura 1. Hospitalizaciones por enfermedad de Kawasaki entre los años 20082017 en niños bajo 5 años de edad.
Con respecto a la asociación temporal entre la circulación de virus respiratorios y hospitalizaciones por EK, entre los años 2010 y 2017 un total de 451 niños bajo 5 años fueron hospitalizados en la RM por EK. Durante este mismo período se registraron 14.902 casos de infecciones virales respiratorias en la red de vigilancia metropolitana en niños de esa misma edad: 6.530 VRS (44\%), 2.570 ADV (17,2\%), 2.307 FLU A (16\%), 1.247 PVI3 (8,4\%), 1.185 MPV (8\%), 596 FLU B (4\%) y 467 PVI1 (3\%). El análisis ecológico de la asociación temporal entre la circulación de virus respiratorios y la incidencia de EK mostró que existe una correlación directa entre PMVRVRS de un mes y año, y PMEK del mes subsiguiente (coeficiente de correlación $+0,441 ; \mathrm{p}<0,001$ ) (Figura 3a); lo mismo ocurre para PMVR-FLU A (coeficiente de correlación +0,362; $\mathrm{p}<0,001$ ) (Figura 3b). PMVR-FLU B y PMVR-MPV presentan igualmente correlaciones directas con PMEK (coeficiente de correlación $+0,443$; $\mathrm{p}<0,001$, y coeficiente de correlación $+0,412 ; \mathrm{p}<0,001$, respectivamente), siendo en el primer caso contemporáneo en mes y año, y el segundo caso con un desfase de un mes entre PMVR y PMEK (Figuras 3c y 3d). Por último, se encontró una correlación negativa entre PMVR-PVI1 y EK0 y EK1 (coeficiente de correlación de -0,381; $\mathrm{p}<0,001$ y $-0,319 ;$ p. 0,002 , respectivamente). No se encontraron correlaciones significativas entre EK y PMVR-ADV y PMVR-PVI3 para todos los escenarios evaluados.

La suma de promedios móviles entre los virus respiratorios (VR) ( $\sum$ PMVR) que mostraron asociación significativa con EK (PMVR-VRS, PMVR-FLU A, PMVR-FLU-B, PMVR-MPV) y su asociación temporal con EK muestran una correlación directa entre $\sum$ PMVR de un mes y año, y PMEK del mismo mes y del mes siguiente (coeficiente de correlación $+0,389 ; \mathrm{p}<0,001$, y coeficiente de correlación $+0,232 ; \mathrm{p}<0,05$, respectivamente; Figuras 3e y $3 \mathrm{f}$ ).

La Tabla 2 muestra los coeficientes de correlación entre los distintos virus respiratorios y EK.

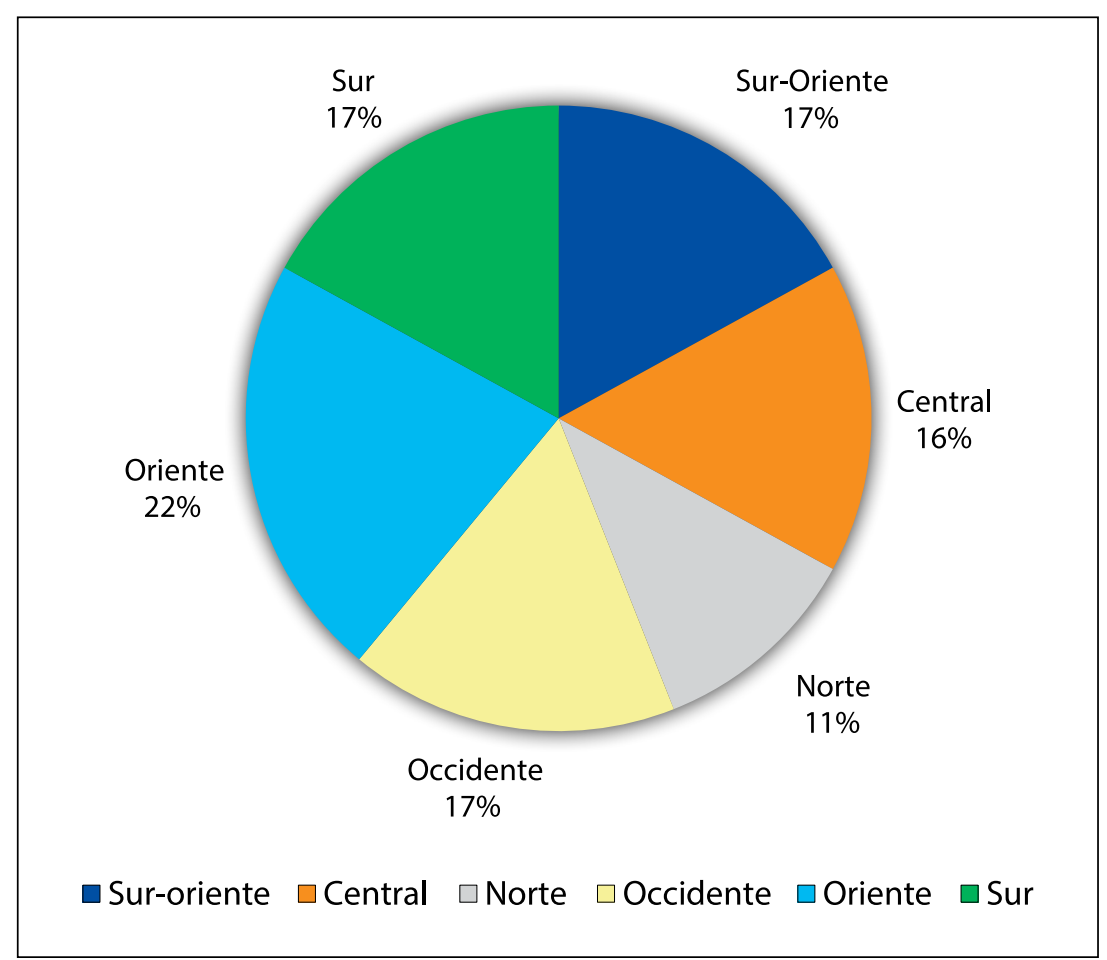

Figura 2. Distribución de casos de enfermedad de Kawasaki en la RM según Servicio de Salud de egreso. 


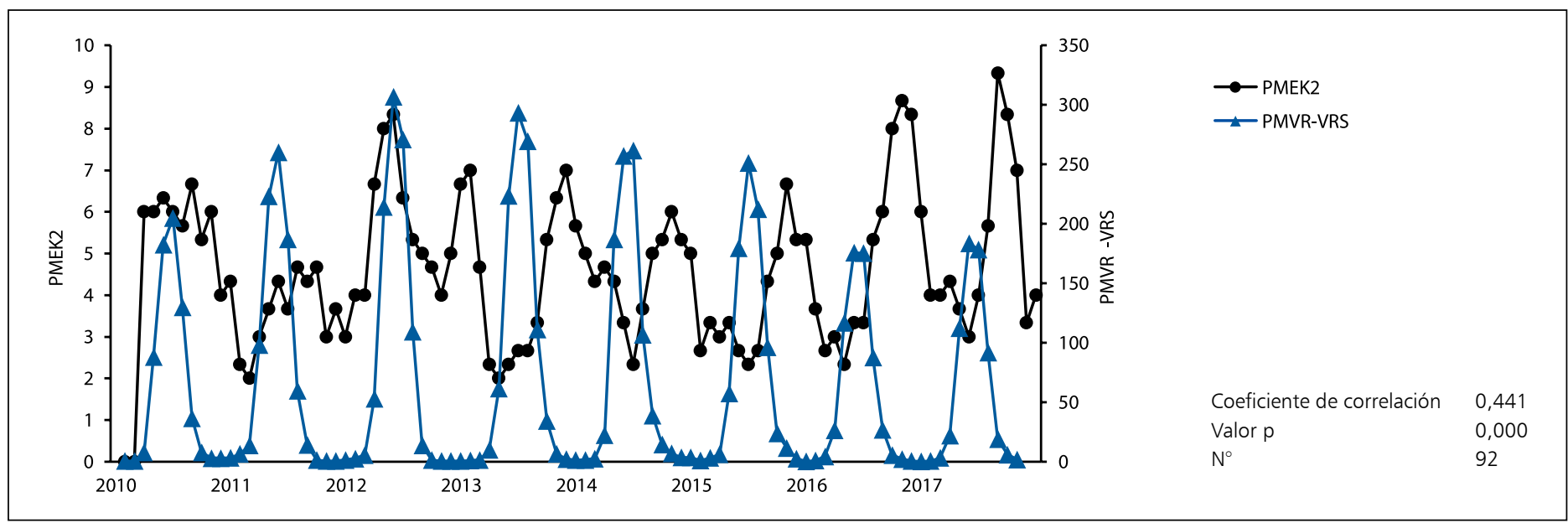

Figura 3a. Correlación entre PMVR-VRS y PMEK2.

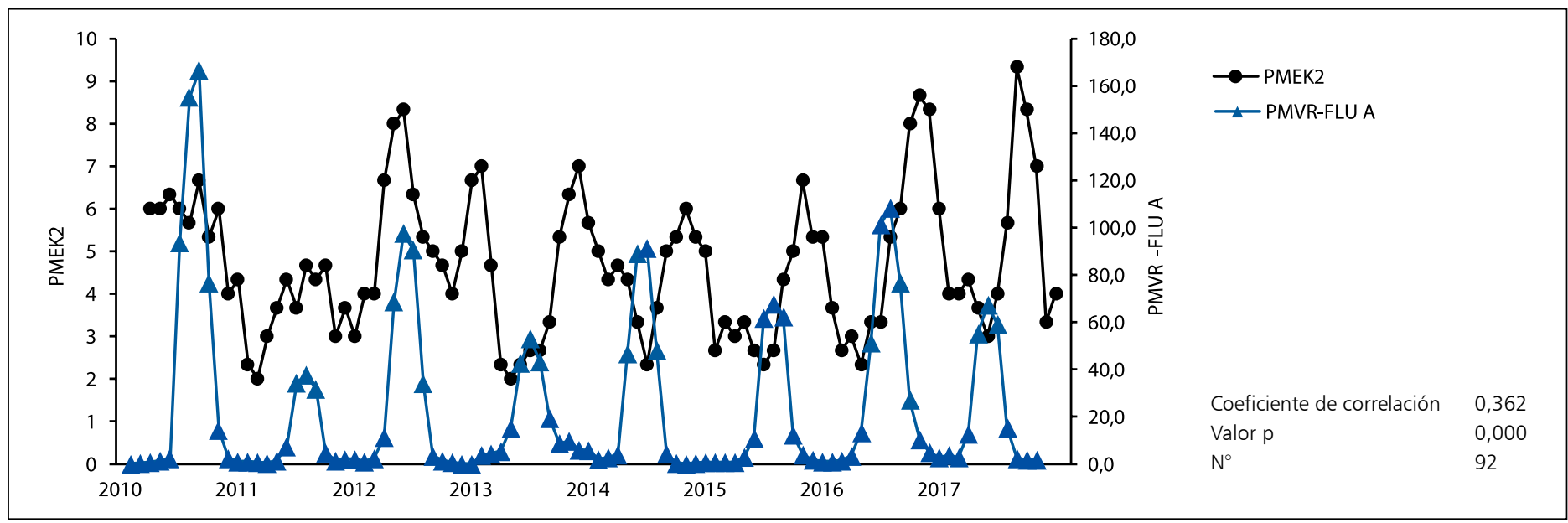

Figura 3b. Correlación entre PMVR-FLU A y PMEK2.

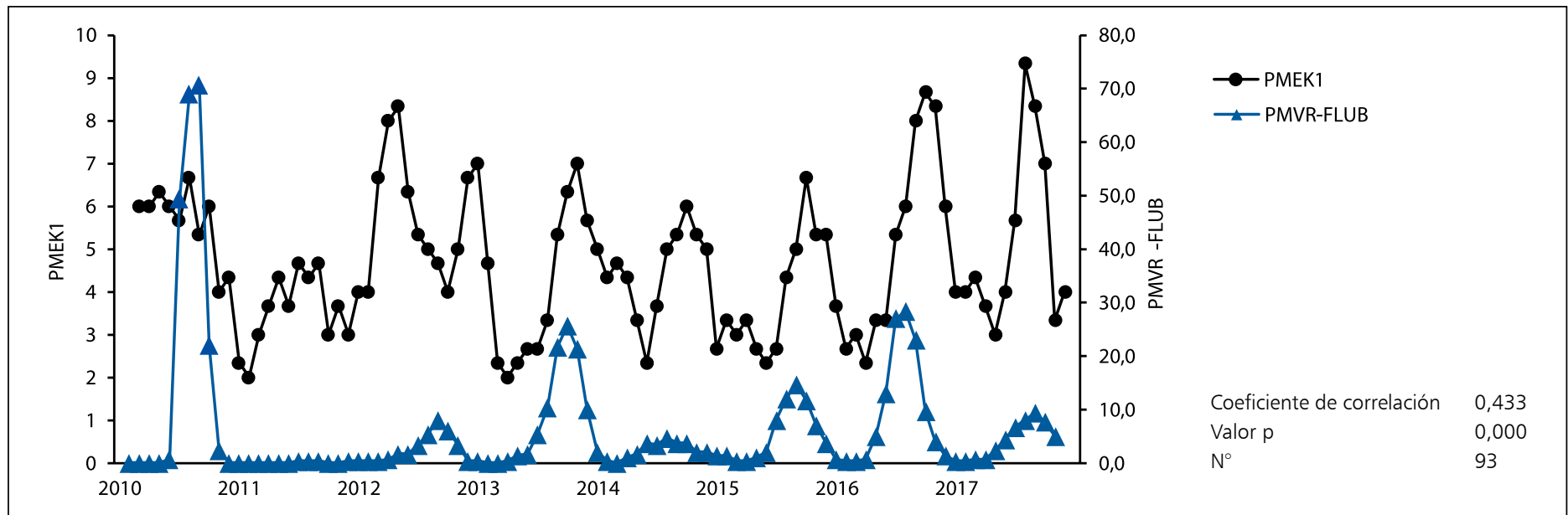

Figura 3c. Correlación entre PMVR-FLU B y PMEK1. 


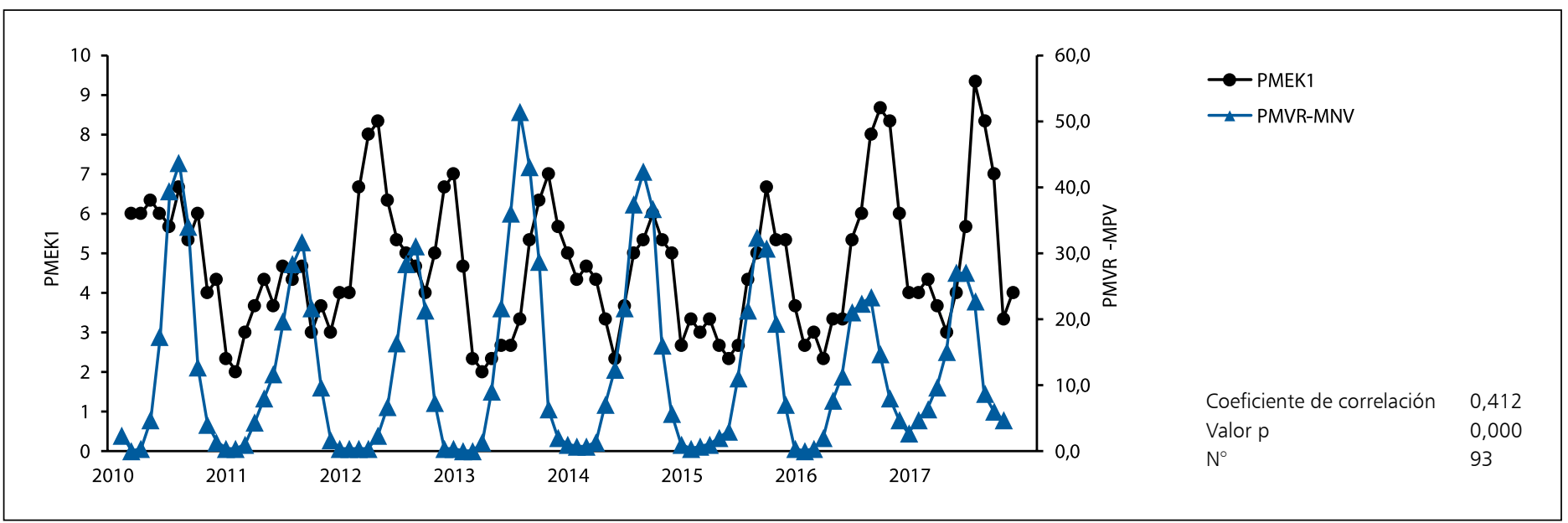

Figura 3d. Correlación entre PMVR-MPV y PMEK1.

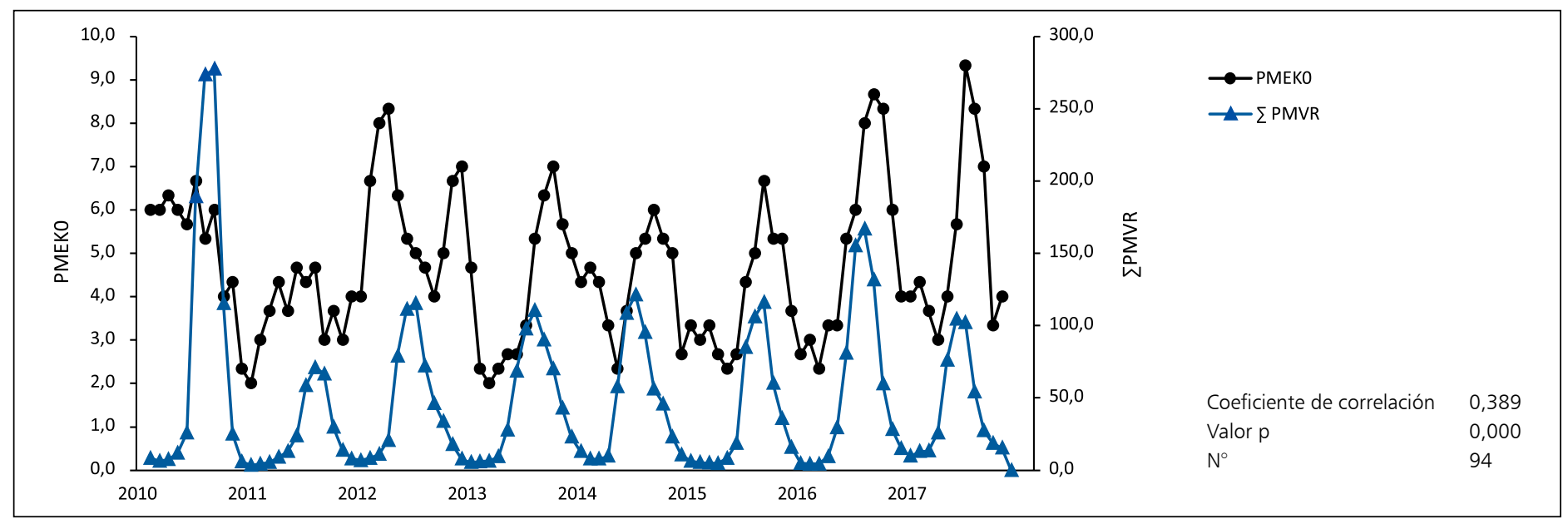

Figura 3e. Correlación entre $\sum$ PMVR y PMEKO.

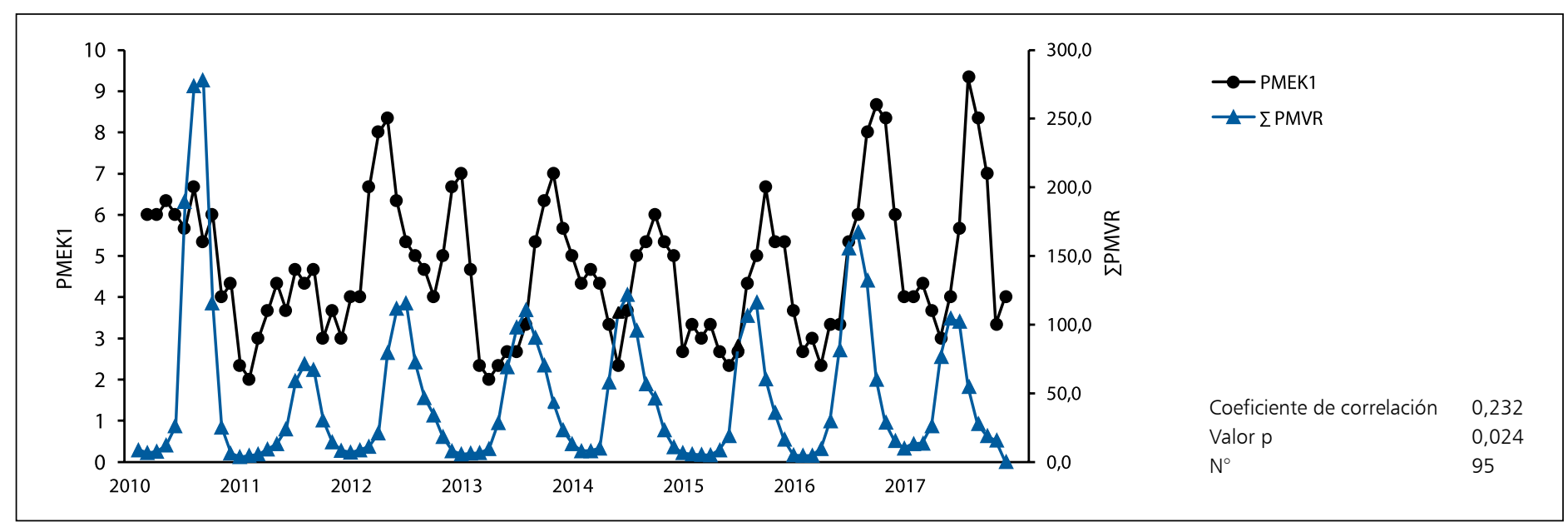

Figura 3f. Correlación entre $\sum$ PMVR y PMEK1. 


\begin{tabular}{|c|c|c|c|}
\hline & EKO* & EK1** & $\mathrm{EK} 2 * * *$ \\
\hline \multicolumn{4}{|l|}{ Virus respiratorio sincicial } \\
\hline Coeficiente de correlación & 0,187 & 0,363 & 0,441 \\
\hline Valor $p^{* * * *}$ & 0,071 & $<0,001$ & $<0,001$ \\
\hline $\mathrm{N}^{\circ}$ & 94 & & \\
\hline \multicolumn{4}{|l|}{ Influenza A } \\
\hline Coeficiente de correlación & 0,251 & 0,359 & 0,362 \\
\hline Valor $\mathrm{p}$ & 0,015 & $<0,001$ & $<0,001$ \\
\hline $\mathrm{N}^{\circ}$ & 94 & 93 & 92 \\
\hline \multicolumn{4}{|l|}{ Adenovirus } \\
\hline Coeficiente de correlación & $-0,138$ & $-0,039$ & $-0,005$ \\
\hline Valor $\mathrm{p}$ & 0,183 & 0,709 & 0,962 \\
\hline $\mathrm{N}^{\circ}$ & 94 & 93 & 92 \\
\hline \multicolumn{4}{|l|}{ Influenza B } \\
\hline Coeficiente de correlación & 0,443 & 0,433 & 0,308 \\
\hline Valor $\mathrm{p}$ & $<0,001$ & $<0,001$ & 0,003 \\
\hline $\mathrm{N}^{\circ}$ & & & 92 \\
\hline \multicolumn{4}{|l|}{ Parainfluenza 1} \\
\hline Coeficiente de correlación & $-0,382$ & $-0,319$ & $-0,197$ \\
\hline Valor $p$ & $<0,001$ & 0,002 & 0,060 \\
\hline $\mathrm{N}^{\circ}$ & & 93 & 92 \\
\hline \multicolumn{4}{|l|}{ Parainfluenza 3} \\
\hline Coeficiente de correlación & $-0,016$ & 0,081 & 0,203 \\
\hline Valor $\mathrm{p}$ & 0,876 & 0,438 & 0,053 \\
\hline $\mathrm{N}^{\circ}$ & 94 & 93 & 92 \\
\hline \multicolumn{4}{|l|}{ Metapneumovirus } \\
\hline Coeficiente de correlación & 0,373 & 0,412 & 0,326 \\
\hline Valor $\mathrm{p}$ & $<0,001$ & $<0,001$ & 0,001 \\
\hline $\mathrm{N}^{\circ}$ & & & 92 \\
\hline \multicolumn{4}{|l|}{$\begin{array}{l}\text { Suma de PMVR ( } 2 \text { PMVR) } \\
\text { (VRS, FLU-A, FLU-B, MPV) }\end{array}$} \\
\hline Coeficiente de correlación & 0,389 & 0,232 & 0,109 \\
\hline Valor $p$ & 0,000 & 0,024 & 0,295 \\
\hline $\mathrm{N}^{\circ}$ & 94 & 95 & 96 \\
\hline \multicolumn{4}{|c|}{$\begin{array}{l}\text { *EKO: correlación entre PMVR y PMEK para un mismo mes y año. }{ }^{* * E K 1: ~ c o r r e l a c i o ́ n ~ e n t r e ~ P M V R ~} \\
\text { de un mes y año, y PMEK del mes siguiente. }{ }^{* * * E K 2: ~ c o r r e l a c i o ́ n ~ e n t r e ~ P M V R ~ d e ~ u n ~ m e s ~ y ~ a n ̃ o, ~} \\
\text { y PMEK del mes subsiguiente. }{ }^{* * *} \text { Valor } p \text { significativo }<0,05 \text {. }\end{array}$} \\
\hline
\end{tabular}

\section{Discusión}

Este trabajo evalúa por primera vez una posible correlación ecológica entre las infecciones respiratorias virales y la epidemiología de la EK en población pediátrica chilena. El análisis de los egresos hospitalarios por EK y la circulación de los virus respiratorios mostró una asociación entre la circulación de VRS, FLU-A, FLU-B y MPV, y las hospitalizaciones por EK concomitantes, o bien con un desfase de uno o dos meses; lo mismo se observa entre la suma de éstos y EK, siendo su asociación no mayor a cada uno por separado. Pese a ser un gran imitador de la enfermedad, no se encontró una correlación significativa entre la incidencia de infecciones por ADV y las hospitalizaciones por EK. Destaca además PVI1 mostrando asociación inversa significativa para EK0 y EK1.

Pese a más de cuatro décadas de estudio, la etiología de la EK es aún desconocida. Algunas líneas de investigación han sugerido como posibles gatillantes a las infecciones virales, basándose en su semejanza clínica con la EK, la marcada estacionalidad de los peak de incidencia de la enfermedad, la edad de presentación, la mala respuesta a tratamiento antimicrobiano $\mathrm{y}$, por último, la alta tasa de detección de virus respiratorios por RPC en pacientes con $\mathrm{EK}^{2}$.

La alta incidencia de EK en grupos étnicos específicos como la población asiática, sugiere que el antecedente de una infección respiratoria viral no es suficiente para el desarrollo de la enfermedad, si no que existiría cierta susceptibilidad genética para desarrollar $\mathrm{EK}^{8,9,13,19}$. Las bases biológicas que sustentan una posible asociación entre EK e infecciones virales son aún materia de estudio. A la fecha se han detectado altos niveles circulantes de interleuquina 1 (IL-1) y una reducción en el número de linfocitos T reguladores en sangre de pacientes con EK, así como un aumento de linfocitos T CD $8+$, importantes para la respuesta antiviral, en autopsias de tejido coronario ${ }^{6,20}$. Se especula que esta respuesta inmune desproporcionada que lleva al desarrollo de la EK ocurriría en pacientes genéticamente predispuestos. El estudio genético de pacientes con EK ha permitido implicar a la fecha a polimorfismos de nucleótido simple en seis genes o regiones genéticas con el desarrollo de la enfermedad: Fc $\gamma$ R2a, $C A S P 3$, HLA-II, ITPKC, CD40 y BLK, principalmente en población asiática ${ }^{4}$. Estos polimorfismos y su importancia como predisponentes al desarrollo de la EK probablemente mostrarán gran variabilidad en distintos grupos étnicos y, por ahora, se necesitan mayores estudios antes de que puedan ser utilizados para la toma de decisiones en la práctica clínica.

La información epidemiológica disponible respecto de la asociación temporal entre virus respiratorios e incidencia de EK es contradictoria. Estudios realizados en el año 2009 en Inglaterra no lograron encontrar una relación temporal entre la incidencia de EK e infecciones por virus respiratorios ${ }^{15}$, así mismo, Kim y cols., en el 2012, no encontraron diferencias entre la prevalencia de infecciones por virus respiratorios en pacientes con EK comparados contra controles sanos ${ }^{11}$. Por el contrario, estudios recientes han reportado una asociación directa entre la incidencia de infecciones por bocavirus y enterovirus y la incidencia de EK en población coreana ${ }^{16}$. En la misma línea, se ha reportado que pacientes con EK tienen una 
Las principales limitaciones de nuestro estudio ecológico se desprenden de las características inherentes al diseño y a las bases de datos utilizadas. La base de egresos hospitalarios utiliza como unidad de análisis las hospitalizaciones y no los pacientes, ya que no se cuenta con un identificador único que permita identificar a un paciente particular; esto impide obtener información en cuanto a re-hospitalizaciones por EK. Otra limitación es que las bases de egresos hospitalarios no consideran pacientes que puedan haber sido diagnosticados y tratados en servicios de urgencia, por ejemplo, durante períodos de contingencia invernal. Con respecto a las limitaciones derivadas del uso de información de la red de vigilancia de los virus respiratorios se debe mencionar que, al no contar con identificadores únicos por paciente, es imposible obtener información respecto de los virus respiratorios detectados exclusivamente en pacientes hospitalizados por EK, con lo que podría haberse realizado un análisis de mayor profundidad acerca de la asociación de infecciones virales respiratorias y $\mathrm{EK}$, aunque en la práctica clínica no necesariamente se encuentra esta relación en la historia clínica. Otra limitación derivada del uso de la información registrada por la red de vigilancia de los virus respiratorios es que el número de virus registrados en esa base de datos es limitada y excluye, por falta de registro, virus respiratorios que fueron analizados en otros estudios con resultados significativos ${ }^{2,16}$. Es importante recalcar que los resultados del presente trabajo establecen asociaciones a nivel ecológico y no relaciones a nivel individual entre virus respiratorios y EK.

En conclusión, el presente estudio ecológico demuestra, de manera ecológica, una correlación temporal directa entre la circulación de VRS, FLU A, FLU B y MPV con EK en niños de Santiago, Chile. Estudios adicionales de base poblacional e individual son necesarios para dilucidar el rol de las infecciones virales en la epidemiología y etiología de la EK.

Agradecimientos. Al Departamento de Enfermedades Infecciosas e Inmunología Pediátrica de la PUC de Chile quienes permitieron la obtención de datos correspondientes a la red de vigilancia de virus respiratorios de la Red de Vigilancia Metropolitana.

\section{Referencias bibliográficas}

1.- Nakamura A, Ikeda K, H K. Aetiological significance of infectious stimuli in Kawasaki disease. Front. Pediatr. 2019 (June 19); 7: article 244. https://doi.org/10.3389/ fped.2019.00244.

2.- Chang L, Lu C, Shao P, Lee P, Lin M, Fan T, et al. Viral infections associated with Kawasaki disease. J. Formos. Med. Assoc. 2014; 113: 148-54 doi: 10.1016/j.jfma.2013.12.008.
3.- Tsai H, Chang L, Lu C, Shao P, Fan T, Cheng A, et al. Transmission of acute infectious illness among cases of Kawasaki disease and their household members. J. Formos. Med. Assoc. 2015; 114: 72-6. doi: 10.1016/j. jfma.2014.07.005.

4.- McCrindle B, Rowley A, Newburger J, Burns J, Bolger A, Gewitz M, et al. Diagnosis, treatment, and long-term management of Kawasaki disease: a scientific statement for health professionals from the
American Heart Association.

Circulation 2017; 135 (17): e927-e999.

https://doi.org/10.1161/

CIR.0000000000000484.

5.- Ritz N. Kawasaki disease: what the paediatrician needs to know. Eur J Pediatr 2017; 176 (8): 1011. doi: 10.1007/s00431-0172943-7.

6.- $\quad$ Son M, B F. Newburger J W. Kawasaki disease. Pediatr. Rev. 2018; 39 (2): 78-90. doi: 10.1542/pir.2016-0182. 
7.- Borzutzky A, Hoyos-Bachiloglu R, Cerda J, Talesnik E. Rising hospitalization rates of Kawasaki disease in Chile between 2001 and 2007. Rheumatol. Int. 2011: 32: 2491-5. doi: 10.1007/s00296-011-2050-4.

8.- Rowley A H, Shulman S T. The epidemiology and pathogenesis of Kawasaki disease. Front. Pediatr. 2018; 6: 374 doi: 10.3389/ fped.2018.00374.

9.- A H Rowley. Is Kawasaki disease an infectious disorder? Int. J. Rheum. Dis. 2017; 21: 20-5. doi: 10.1111/1756-185X.13213.

10.- Hoyos-Bachiloglu R, García A, Morales P, Cerda J, Talesnik E, B A. Distribución geográfica de la enfermedad de Kawasaki en Chile. Rev Chilena Infectol 2016; 33; 12-8 http://dx.doi.org/10.4067/S071610182016000100002

11.- Kim J H, Jin Yu J, Lee J, Kim M N, Ki Ko H, Soon Choi H, et al. Detection rate and clinical impact of respiratory viruses in children with Kawasaki disease. Korean J Pediatr 2012; 55 (12): 470-3. doi: https://doi.org/10.3345/ kjp.2012.55.12.470.

12.- Rowley A H, Baker S, Shulman S, Rand K, Tretiakova S, Perlman E, et al. Ultrastructural, immunofluorescence, and RNA evidence support the hypothesis of a "new" virus associated with Kawasaki disease. J Infect Dis. 2011; 203: 1021-30. doi: 10.1093/infdis/jiq136

13.- Nagata S. Causes of Kawasaki disease - From past to present. Front. Pediatr. $2019 \mathrm{Feb} ; 7: 18$ doi: 10.3389/fped.2019.00018.

14.- Wu M, Nakamura Y, Burns J, Rowley A, Takahashi K, Newburger J, et al. State-ofthe-art basic and clinical science of Kawasaki disease. Pediatr. Health 2008; 2 (4): 405-9. doi: 10.2217/17455111.2.4.405.

15.- Harnden A, Mayon-White R, Perera R, Yeates D, Goldacre M, Burgner D. Kawasaki disease in England: ethnicity, deprivation, and respiratory pathogens. Pediatr. Infect. Dis. J. 2009; 28: 21-4. doi: 10.1097/ inf.0b013e3181812ca4.

16.- Kim G B, Park S, Kwon B S, Han J W, Park Y W, Hong Y M. Evaluation of the temporal association between Kawasaki disease and viral infections in South Korea. Korean Circ. J. 2014; 44: 250-4. doi: 10.4070/ kcj.2014.44.4.250.

17.- Cavallo F, Chiarelli F. An outbreak of Kawasaki-like disease in children during
SARS-COV- 2 epidemic: no surprise? Acta Biomed 2020; 91 (3): e2020015. doi: 10.23750/ abm.v91i3.10305.

18.- Berardicurti O, Conforti A, Ruscitti P, Cipriani P, Giacomelli R. The wide spectrum of Kawasaki-like disease associated with SARS-CoV-2 infection. Expert Rev. Clin. Immunol. 2020 Dec; 16 (12): 1205-15. doi: 10.1080/1744666X.2021.1847643.

19. Turnier J, Anderson M, Heizer H, Jone P, Glode M, D S. Concurrent respiratory viruses and Kawasaki disease. Pediatr. Rev. 2015; 136: e609-e614. doi: 10.1542/peds.20150950 .

20. van Stijn D, Burgner D, Levin M, Kuipers IM, Hutten BA, et al. Dissecting Kawasaki disease: a state-of-the-art review. Eur J Pediatr 2017 Aug; 176 (8): 995-1009. doi: 10.1007/s00431017-2937-5.

21.- Jorquera H, Borzutzky A, Hoyos-Bachiloglu R, García A. Association of Kawasaki disease with tropospheric winds in Central Chile: is wind-borne desert dust a risk factor? Environ. Int. 2015; 78: 32-8 doi: 10.1016/j. envint.2015.02.007. 